\title{
Sequent Calculi for the Modal $\mu$-Calculus over S5
}

\author{
LUCA ALBERUCCI, University of Berne, Switzerland. \\ E-mail: albe@iam.unibe.ch
}

\begin{abstract}
We present two sequent calculi for the modal $\mu$-calculus over S5 and prove their completeness by using classical methods. One sequent calculus has an analytical cut rule and could be used for a decision procedure the other uses a modified version of the induction rule. We also provide a completeness theorem for Kozen's Axiomatization over S5 without using the completeness result established by Walukiewicz for the modal $\mu$-calculus over arbitrary models.
\end{abstract}

Keywords: Modal $\mu$-calculus, modal logic, proof-theory, sequent calculus, completeness.

\section{Introduction}

Modal $\mu$-calculus is an extension of modal logic with least and greatest fixpoint constructors and allows us to study fixpoints, which play an important role as extensions for many modal logics, on a sufficiently abstract level.

The expression ' $\mu$-calculus' combined with the idea to introduce fixpoint constructors to monotonic functions on complete lattices was first introduced by Scott and De Bakker (unpublished data). The book of Arnold and Niwinski [3] provides a good overview over this general notion of $\mu$-calculus. Modal $\mu$-calculus can be seen as a special case where we restrict ourselves to the complete lattice given by the powerset of states of a transition system. It was introduced by Kozen in his seminal work [7]. There, also the axiomatization $\mathrm{Koz}$ is introduced which is basically the extension of minimal modal logic $\mathrm{K}$ with the so-called Park fixpoint induction principles. Kozen himself could prove completeness for the aconjunctive fragment but failed for the full language. Full completeness was established by Walukiewicz in [10], the proof is very involved and strongly relies on methods from automata theory and infinite games.

For proof-theorists induction principles in a modal context represent a big challenge and are quite difficult to handle in a pure syntactical manner. Therefore, proof-theoretical research on the modal $\mu$-calculus has concentrated on, mainly infinitary, systems different from Koz (see e.g. [6, 8] and [5]). One aim of our work is to study proof-theoretical fixpoints and induction on S5-models. In order to do this we present two sequent calculi, $\mathrm{T}_{\mathrm{S} 5^{\mu}}^{1}$ and $\mathrm{T}_{\mathrm{S} 5^{\mu}}^{2}$, for the modal $\mu$-calculus over S5.

The first calculus, $\mathrm{T}_{\mathrm{S} 5^{\mu}}^{1}$, uses a modified induction rule, compared with the one used in Kozen's Axiomatization. We show its correctness and, by working exclusively syntactically in the calculus, that for formulae in a certain normal form $\mathrm{T}_{\mathrm{S} 5^{\mu}}^{1}$ proves that the fixpoint is reached after two iterations. This result has first been proved in the joint work with Facchini [2] by using game-theoretical methods and the correspondence of parity games with modal $\mu$-calculus. Then, we show completeness of the second system, $\mathrm{T}_{\mathrm{S} 5^{\mu}}^{2}$, by using a canonical model construction. The calculus $\mathrm{T}_{\mathrm{S} 5^{\mu}}^{2}$ only uses an analytical cut rule and, therefore, could provide a decision procedure for validity. By embedding $\mathrm{T}_{\mathrm{S} 5^{\mu}}^{1}$ into $\mathrm{T}_{\mathrm{S} 5^{\mu}}^{2}$ we get completeness and correctness for both calculi. 


\section{Sequent Calculi for the Modal $\mu$-Calculus over S5}

Finally, we show the completeness for Kozen's Axiomatization over S5, Koz ${ }^{\mathrm{S}}$. The main ingredient of the completeness proof is the fact that for formulae in normal form the fixpoint is reached after two iterations and that $\mathrm{Koz}^{\mathrm{S} 5}$ proves the equivalence of a formula with its normal form. Our completeness proof does not use the completeness result of Walukiewicz over arbitrary structures.

In the next section we introduce the modal $\mu$-calculus. In Section 3, we define the calculi $\mathrm{T}_{\mathrm{S} 5}^{1}$ and $\mathrm{T}_{\mathrm{S} 5^{\mu}}^{2}$. Section 4 is devoted to $\mathrm{T}_{\mathrm{S} 5^{\mu}}^{1}$ and Section 5 to $\mathrm{T}_{\mathrm{S} 5^{\mu}}^{2}$. In Section 6, we embed $\mathrm{T}_{\mathrm{S} 5^{\mu}}^{1}$ in $\mathrm{T}_{\mathrm{S} 5^{\mu}}^{2}$ and prove their completeness and correctness. We conclude by showing completeness of $\mathrm{Koz}^{\mathrm{S} 5}$.

\section{The propositional modal $\mu$-calculus}

\subsection{Syntax}

The language of the modal $\mu$-calculus results by adding greatest and least fixpoint operators to propositional modal logic. More precisely, given a countable infinite set $\mathrm{P}$ of propositional variables, the collection, $\mathcal{L}_{\mu}$, of modal $\mu$-formulae (or simply $\mu$-formulae) is defined as follows:

$$
\varphi::=p|\sim p| \top|\perp|(\varphi \wedge \varphi)|(\varphi \vee \varphi)| \diamond \varphi|\square \varphi| \mu x . \varphi \mid v x . \varphi
$$

where $p, x \in \mathrm{P}$ and $x$ occurs only positively in $\sigma x . \varphi(\sigma \in\{v, \mu\})$, i.e. $\sim x$ is not a subformula of $\varphi$. $\mathcal{L}_{\bmod }$ denotes the pure modal fragment of $\mathcal{L}_{\mu}$.

The fixpoint operators $\mu$ and $v$ can be viewed as quantifiers. Therefore we use the standard terminology and notations as for quantifiers and, for instance, free $(\varphi)$ denotes the set of all propositional variables occurring free in $\varphi$ and bound $(\varphi)$ those occurring bound. By renaming bound variables we can achieve that bound and free variables are distinct. If nothing else mentionned we assume that this is the case. If $\psi$ is a subformula of $\varphi$, we write $\psi \leq \varphi$. We write $\psi<\varphi$ when $\psi$ is a proper subformula.

Let $\varphi(x)$ and $\psi$ be two $\mu$-formulae. The substitution of all occurrences of $x$ with $\psi$ in $\varphi$ is denoted by $\varphi[x / \psi]$ or sometimes simply $\varphi(\psi)$. Simultaneous substitution of all $x_{i}$ by $\psi_{i}(i \in\{1, \ldots, n\})$ is denoted by $\varphi\left[x_{1} / \psi_{1}, \ldots, x_{n} / \psi_{n}\right]$. If $\Gamma$ is the set of formulae $\left\{\alpha_{1}, \alpha_{2}, \ldots\right\}$ then $\Gamma[x / \psi]$ denotes the set $\left\{\alpha_{1}[x / \psi], \alpha_{2}[x / \psi], \ldots\right\}$. For a formal introduction of substitution we refer to Alberucci [1].

The negation $\neg \varphi$ of a $\mu$-formula $\varphi$ is defined inductively such that $\neg p \equiv \sim p$ and $\neg(\sim p) \equiv p$, by using de Morgan dualities for boolean connectives and the usual modal dualities for $\diamond$ and $\square$. For $\mu, \nu$ we define

$$
\neg \mu x . \varphi(x) \equiv v x . \neg \varphi(x)[x / \neg x] \quad \text { and } \quad \neg v x . \varphi(x) \equiv \mu x . \neg \varphi(x)[x / \neg x] .
$$

As usual, we introduce implication $\varphi \rightarrow \psi$ as $\neg \varphi \vee \psi$ and equivalence $\varphi \leftrightarrow \psi$ as $(\varphi \rightarrow \psi) \wedge(\varphi \rightarrow \psi)$.

If $x \leq \varphi$ and $x$ is in the scope of a $\diamond$ or in the scope of a $\square$ operator, then we say that $x$ is guarded in $\varphi$. A formula $\varphi$ of $\mathcal{L}_{\mu}$ is said to be guarded if for every subformula of $\varphi$ of the form $\sigma x . \alpha(\sigma \in\{\mu, \nu\})$, $x$ is guarded in $\alpha$. Let $\varphi(x)$ be a $\mu$-formula. If $x$ is free and occurs only positively in $\varphi$, then we define $\varphi^{n}(x)$ for all $n$ inductively such that $\varphi^{1}(x)=\varphi$ and such that

$$
\varphi^{k+1}(x) \equiv \varphi\left[x / \varphi^{k}(x)\right] .
$$

We define $\varphi^{n}(\perp)$ as $\varphi^{n}(x)[x / \perp]$ and $\varphi^{n}(\top)$ as $\varphi^{n}(x)[x / \top]$. 
In the joint work with Alberucci and Krähenbühl (manuscript in preparation) (see also [1]) we show that there exists a measure for the syntactical complexity of formulae, $\operatorname{rank}(\varphi)$, which assigns to each formula $\varphi$ an ordinal number such that the following holds:

- $\operatorname{rank}(p)=\operatorname{rank}(\sim p)=\operatorname{rank}(\top)=\operatorname{rank}(\perp)=1$,

- $\operatorname{rank}(\triangle \alpha)=\operatorname{rank}(\alpha)+1$ where $\Delta \in\{\square, \diamond\}$,

- $\operatorname{rank}(\alpha \circ \beta)=\max \{\operatorname{rank}(\alpha), \operatorname{rank}(\beta)\}+1$ where $\circ \in\{\wedge, \vee\}$,

- $\operatorname{rank}(\sigma x . \alpha)=\sup \left\{\operatorname{rank}\left(\alpha^{n}(x)\right)+1 ; n \in \mathbb{N}\right\}$ where $\sigma \in\{\nu, \mu\}$.

It is an easy exercise to show that for all formulae $\varphi$ we have that $\operatorname{rank}(\varphi)=\operatorname{rank}(\neg \varphi)$.

We say that a formula $\varphi$ well-bounded if for all subformulae of the form $\sigma x . \alpha(\sigma \in\{\mu, \nu\})$ we have that $x$ appears free at most once in $\alpha$. By replacing all subformulae $\sigma x . \alpha(x, \ldots, x)$ of $\varphi$ by $\sigma x_{1} \ldots \sigma x_{n} . \alpha\left(x_{1}, \ldots, x_{n}\right)$, where $x_{1}, \ldots, x_{n}$ are new variables and $\sigma \in\{\mu, \nu\}$, we can convert $\varphi$ to a well-bounded formula $\mathrm{wb}(\varphi)$.

LEMMA 2.1

For formulae $\varphi$ such that $x$ appears only positively we have that if $\varphi$ is well-bounded then for all $n \in \mathbb{N}$ the formula $\varphi^{n}(x)$ is well-bounded, too.

PROOF. Follows from the fact that for all $n \in \mathbb{N}$ no variable gets newly bound by the substitution $\varphi\left[x / \varphi^{n}(\top)\right]$. Therefore, for all subformulae of $\varphi\left[x / \varphi^{n}(\top)\right]$ of the form $\sigma x . \alpha$ we have that $x$ appears at most once free in $\alpha$.

Kozen's Axiomatization, Koz, is a Hilbert-style axiomatization and consists of the following axioms and rules.

AxıOMS

Koz contains all axioms of the classical propositional calculus, the distribution axiom

$$
\square(\varphi \rightarrow \psi) \rightarrow(\square \varphi \rightarrow \square \psi)
$$

and the fixpoint axiom

$$
v x . \varphi \leftrightarrow \varphi(v x . \varphi)
$$

INFERENCE RULES

In addition to the classical Modus Ponens (MP) we have the Necessitation Rule (Nec) from modal logic.

$$
\frac{\varphi \quad \varphi \rightarrow \psi}{\psi}(\mathrm{MP}) \quad \frac{\varphi}{\square \varphi}(\mathrm{Nec})
$$

Further, for any formula $\varphi(x)$ such that $x$ appears only positively we have the Induction Rule (ind) to handle fixpoints.

$$
\frac{\psi \rightarrow \varphi(\psi)}{\psi \rightarrow v x . \varphi} \text { (ind) }
$$

Kozen's Axiomatization over $\mathrm{S} 5, \mathrm{Koz}^{\mathrm{S} 5}$, consists of the axioms and inference rules of $\mathrm{Koz}$ and additionally of the S5 axiom schemes

$\mathrm{T}: \square \varphi \rightarrow \varphi$,

4: $\square \varphi \rightarrow \square \square \varphi$, and

5: $\diamond \varphi \rightarrow \square \diamond \varphi$.

We write $\mathrm{Koz}^{\mathrm{S} 5} \vdash \varphi$ if $\varphi$ is provable in $\mathrm{Koz}^{\mathrm{S} 5}$. S5 is obtained from $\mathrm{Koz}^{\mathrm{S} 5}$ by omitting induction and fixpoint axioms. 


\section{Sequent Calculi for the Modal $\mu$-Calculus over S5}

\subsection{Semantics}

The semantics of modal $\mu$-calculus is given by transition systems. A transition system $\mathcal{T}$ is of the form $\left(\mathrm{S}, \rightarrow^{\mathcal{T}}, \lambda^{\mathcal{T}}\right)$ where $\mathrm{S}$ is a set of states, $\rightarrow^{\mathcal{T}}$ is a binary relation on $\mathrm{S}$ called the accessibility relation and $\lambda: \mathrm{P} \rightarrow \wp(\mathrm{S})$ is a valuation for all propositional variables. In this article, we concentrate on transition systems whose accessibility relation is an equivalence relation, i.e. reflexive, transitive and symmetric. It is the class of all S5-models.

Let $\lambda$ be a valuation, $p$ a propositional variable and $\mathrm{S}^{\prime}$ a subset of states $S$; we set for all propositional variables $p^{\prime}$

$$
\lambda\left[p \mapsto \mathrm{S}^{\prime}\right]\left(p^{\prime}\right)= \begin{cases}\mathrm{S}^{\prime} & \text { if } p^{\prime}=p, \\ \lambda\left(p^{\prime}\right) & \text { otherwise }\end{cases}
$$

Given a transition system $\mathcal{T}=\left(\mathrm{S}, \rightarrow^{\mathcal{T}}, \lambda^{\mathcal{T}}\right)$, then $\mathcal{T}\left[p \mapsto \mathrm{S}^{\prime}\right]$ denotes the transition system $\left(\mathrm{S}, \rightarrow^{\mathcal{T}}\right.$, $\left.\lambda^{\mathcal{T}}\left[p \mapsto \mathrm{S}^{\prime}\right]\right)$. Given a transition system $\mathcal{T}$, the denotation of $\varphi$ in $\mathcal{T},\|\varphi\|_{\mathcal{T}}$, i.e. the set of states satisfying a formula $\varphi$ is defined inductively on the structure of $\varphi$. Simultaneously for all transition systems we set

- $\|p\|_{\mathcal{T}}=\lambda(p)$ and $\|\sim p\|_{\mathcal{T}}=\lambda(p)$ for all $p \in \mathrm{P}$,

- $\|\alpha \wedge \beta\|_{\mathcal{T}}=\|\alpha\|_{\mathcal{T}} \cap\|\beta\|_{\mathcal{T}}$ and $\|\alpha \vee \beta\|_{\mathcal{T}}=\|\alpha\|_{\mathcal{T}} \cup\|\beta\|_{\mathcal{T}}$,

- $\|\square \alpha\|_{\mathcal{T}}=\left\{s \in \mathrm{S} \mid \forall t\left(\left(s \rightarrow_{\mathcal{T}}^{\mathcal{T}} t\right) \Rightarrow t \in\|\alpha\|_{\mathcal{T}}\right)\right\}$,

- $\|\triangleright \alpha\|_{\mathcal{T}}=\left\{s \in \mathrm{S} \mid \exists t\left(\left(s \rightarrow^{\mathcal{T}} t\right) \wedge t \in\|\alpha\|_{\mathcal{T}}\right)\right\}$,

- $\|v x . \alpha\|_{\mathcal{T}}=\bigcup\left\{\mathrm{S}^{\prime} \subseteq \mathrm{S} \mid \mathrm{S}^{\prime} \subseteq\|\alpha(x)\|_{\mathcal{T}\left[x \mapsto \mathrm{S}^{\prime}\right]}\right\}$ and

- $\|\mu x . \alpha\|_{\mathcal{T}}=\bigcap\left\{\mathrm{S}^{\prime} \subseteq \mathrm{S} \mid\|\alpha(x)\|_{\mathcal{T}\left[x \mapsto \mathrm{S}^{\prime}\right]} \subseteq \mathrm{S}^{\prime}\right\}$.

If $s \in\|\varphi\|_{\mathcal{T}}$ then we say that $\varphi$ is valid in $s$ and write $s=\mathcal{T} \varphi$ or when clear from the context simply $s=\varphi$. An easy induction shows that $s=\varphi$ if and only if $s \not \models \neg \varphi$. A formula $\varphi$ is valid in $\mathcal{T}$ if it is valid in all states of $\mathcal{T}$. We then write $\mathcal{T} \models \varphi . \varphi$ is valid if it is valid in all S5 models. We then write $\models$ S5 $\varphi$. For any finite set of formulae $\Gamma$ we write $s \models \Gamma$ if we have $s=\bigvee \Gamma$, analogously for $\mathcal{T} \models \Gamma$ and $=\mathrm{S} 5 \Gamma$.

For a formula $\varphi(x)$ and set of states $\mathrm{S}^{\prime} \subseteq \mathrm{S}$ we sometimes write $\left\|\varphi\left(\mathrm{S}^{\prime}\right)\right\|_{\mathcal{T}}$ instead of $\|\varphi(x)\|_{\mathcal{T}\left[x \mapsto \mathrm{S}^{\prime}\right]}$. When clear from the context we use $\|\varphi(x)\|_{\mathcal{T}}$ for the function

$$
\|\varphi(x)\|_{\mathcal{T}}:\left\{\begin{array}{l}
\wp(\mathrm{S}) \rightarrow \wp(\mathrm{S}) \\
\mathrm{S}^{\prime} \mapsto\left\|\varphi\left(\mathrm{S}^{\prime}\right)\right\|_{\mathcal{T}} .
\end{array}\right.
$$

By the well-known Tarski-Knaster Theorem, cf. [9], $\|v x . \alpha(x)\|_{\mathcal{T}}$ is the greatest fixpoint and $\|\mu x . \alpha(x)\|_{\mathcal{T}}$ the least fixpoint of the operator $\|\alpha(x)\|_{\mathcal{T}}$, we have that

$$
\|v x . \alpha(x)\|_{\mathcal{T}}=\operatorname{GFP}\left(\|\alpha(x)\|_{\mathcal{T}}\right) \quad \text { and } \quad\|\mu x . \alpha(x)\|_{\mathcal{T}}=\operatorname{LFP}\left(\|\alpha(x)\|_{\mathcal{T}}\right)
$$

Further, by Tarski-Knaster Theorem we also have that

$$
\|v x . \alpha(x)\|_{\mathcal{T}}=\|\neg \mu x . \neg \alpha[x / \neg x]\|_{\mathcal{T}} \quad \text { and } \quad\|\mu x . \alpha(x)\|_{\mathcal{T}}=\|\neg v x . \neg \alpha[x / \neg x]\|_{\mathcal{T}} .
$$

Using this result with an easy induction we can verify that negation is well-defined in the sense that for any state $s$ in a transition system $\mathcal{T}$ and any formula $\varphi$ we have that

$$
s \models \mathcal{T} \varphi \quad \text { if and only if } \quad s \not \models \mathcal{T} \neg \varphi .
$$


Part (1) of the following proposition is the correctness of $\mathrm{Koz}^{\mathrm{S} 5}$ is a straightforward induction on the length of the derivation, part (2) is a straightforward consequence of the completeness of S5 (see e.g. [4]).

PROPOSITION 2.2

(1) For all formulae $\varphi \in \mathcal{L}_{\mu}$ we have that

$$
\mathrm{Koz}^{\mathrm{S} 5} \vdash \varphi \Rightarrow \models \mathrm{S} 5 \varphi
$$

(2) For all formulae $\varphi \in \mathcal{L}_{\text {mod }}$ we have that

$$
\mathrm{S} 5 \vdash \varphi \Leftrightarrow \mathrm{Koz}^{\mathrm{S} 5} \vdash \varphi \Leftrightarrow \models \mathrm{S} 5 \varphi .
$$

\section{Introducing the sequent calculi $\mathrm{T}_{\mathrm{S}{ }^{\mu}}^{1}$ and $\mathrm{T}_{\mathrm{S}{ }^{\mu}}^{2}$}

In this section, we introduce the Tait-style sequent calculi $\mathrm{T}_{\mathrm{S} 5^{\mu}}^{1}$ and $\mathrm{T}_{\mathrm{S} 5^{\mu}}^{2}$. Our sequents are sets of formulae denoted by major Greek letters, $\Gamma, \Delta, \Sigma$, etc. Given a sequent $\Gamma$ by $\square \Gamma$ we denote the sequent $\{\square \alpha ; \alpha \in \Gamma\}$ and analogously for $\diamond \Gamma$ and $\neg \Gamma$.

First, for all sets of formulae $\Gamma$ we define $\operatorname{sub}(\Gamma)$ to be the smallest set such that $\Gamma \subseteq \operatorname{sub}(\Gamma)$ and such that

- if $\alpha \wedge \beta, \alpha \vee \beta \in \operatorname{sub}(\Gamma)$ then $\alpha, \beta \in \operatorname{sub}(\Gamma)$,

- if $\square \alpha, \diamond \alpha, \neg \alpha \in \operatorname{sub}(\Gamma)$ then $\alpha \in \operatorname{sub}(\Gamma)$,

- if $x$ appears at most once and guarded in $\alpha$ then $\mu x . \alpha \in \operatorname{sub}(\Gamma)$ implies that $\alpha^{2}(\perp) \in \operatorname{sub}(\Gamma)$, and $v x . \alpha \in \operatorname{sub}(\Gamma)$ implies that $\alpha^{2}(\top) \in \operatorname{sub}(\Gamma)$,

- if $x$ appears at most once and not guarded in $\alpha$ then $\mu x . \alpha \in \operatorname{sub}(\Gamma)$ implies that $\alpha(\perp) \in \operatorname{sub}(\Gamma)$, and $v x . \alpha \in \operatorname{sub}(\Gamma)$ implies that $\alpha(T) \in \operatorname{sub}(\Gamma)$.

Note, that by definition we have that $\operatorname{sub}(\Gamma)=\bigcup_{\varphi \in \Gamma} \operatorname{sub}(\varphi)$. And therefore, by induction on rank $(\varphi)$ we can show that if $\Gamma$ is finite then $\operatorname{sub}(\Gamma)$ is finite, too. The closure of $\Gamma, \mathcal{C}(\Gamma)$, is defined as the following set

$$
\begin{gathered}
\operatorname{sub}(\Gamma) \cup\{\square \alpha ; \alpha \in \operatorname{sub}(\Gamma) \text { and } \alpha \text { not of the form } \square \beta \text { or } \diamond \beta\} \cup \ldots \\
\ldots \cup\{\diamond \alpha ; \alpha \in \operatorname{sub}(\Gamma) \text { and } \alpha \text { not of the form } \square \beta \text { or } \diamond \beta\} .
\end{gathered}
$$

We have that if $\Gamma$ is finite then $\mathcal{C}(\Gamma)$ is also finite and that $\alpha \in \mathcal{C}(\Gamma)$ if and only if $\neg \alpha \in \mathcal{C}(\Gamma)$. Further, by using Lemma 2.1 we have the following lemma.

\section{LEMMA 3.1}

If all formulae $\varphi \in \Gamma$ are well-bounded then we have that all formulae in $\mathcal{C}(\Gamma)$ are well-bounded.

In the following we present the relevant Tait-style inference rules.

$$
\begin{gathered}
\frac{\Gamma, \nu x . \varphi, \neg v x . \varphi}{\Gamma\left(\mathrm{Ax}^{\nu}\right)} \underset{\Gamma, p, \sim p}{(\mathrm{Ax})} \quad \frac{\Gamma, \alpha \quad \Gamma, \beta}{\Gamma, \alpha \wedge \beta}(\wedge) \quad \frac{\Gamma, \alpha, \beta}{\Gamma, \alpha \vee \beta}(\vee) \\
\frac{\diamond \Delta, \square \Gamma, \alpha}{\diamond \Delta, \square \Gamma, \square \alpha, \Sigma}(\square) \quad \frac{\Gamma, \varphi}{\Gamma, \diamond \varphi}(\diamond)
\end{gathered}
$$




$$
\left.\frac{\Gamma, \varphi(\mu x . \varphi)}{\Gamma, \mu x . \varphi}\left(\text { unf }^{\mu}\right) \quad \frac{\Gamma, \varphi(v x . \varphi)}{\Gamma, v x . \varphi}\left(\text { unf }^{v}\right) \quad \frac{\diamond \Delta, \square \Gamma, \neg \varphi, \alpha(\varphi)}{\diamond \Delta, \square \Gamma, \neg \varphi, v x . \alpha, \Sigma} \text { (ind }^{+}\right)
$$

If $x$ appears at most once and guarded in $\alpha(x)$ :

$$
\frac{\Gamma, \alpha^{2}(\top)}{\Gamma, v x . \alpha}\left(v^{2}\right) \quad \frac{\Gamma, \alpha^{2}(\perp)}{\Gamma, \mu x . \alpha}\left(\mu^{2}\right)
$$

If $x$ appears at most once and not guarded in $\alpha(x)$ :

$$
\begin{gathered}
\frac{\Gamma, \alpha(\top)}{\Gamma, v x . \alpha}(v) \quad \frac{\Gamma, \alpha(\perp)}{\Gamma, \mu x . \alpha}(\mu) \\
\frac{\Gamma, \alpha \quad \Delta, \neg \alpha}{\Gamma, \Delta} \text { (cut) } \frac{\Gamma, \alpha \quad \Delta, \neg \alpha}{\Gamma, \Delta} \text { (Ccut) where } \alpha \in \mathcal{C}(\Gamma, \Delta) .
\end{gathered}
$$

DEFINITION 3.2

The systems $\mathrm{T}_{\mathrm{S} 5^{\mu}}^{1}$ and $\mathrm{T}_{\mathrm{S} 5^{\mu}}^{2}$ are defined by the following rule schemes

- $\mathrm{T}_{\mathrm{S} 5^{\mu}}^{1}:(\mathrm{Ax}),\left(\mathrm{Ax}^{\nu}\right),(\wedge),(\vee),(\square),(\diamond),\left(\right.$ ind $\left.^{+}\right),\left(\right.$unf $\left.^{\mu}\right),\left(\mathrm{unf}^{\nu}\right),(\mathrm{cut})$.

- $\mathrm{T}_{\mathrm{S}^{\mu}}^{2}:(\mathrm{Ax}),(\wedge),(\vee),(\square),(\diamond),(\mu),(\nu),\left(v^{2}\right),\left(\mu^{2}\right),(\mathcal{C}$ cut $)$.

We write $\mathrm{T}_{\mathrm{S} 5^{\mu}}^{1} \vdash \Gamma$ if there is a proof of $\Gamma$ in $\mathrm{T}_{\mathrm{S} 5^{\mu}}^{1}, \mathrm{~T}_{\mathrm{S} 5^{\mu}}^{1} \vdash^{n} \Gamma$ if the proof has length (depth of the proof tree) at most $n$, and we write $\mathrm{T}_{S 5^{\mu}}^{1} \vdash^{<n} \Gamma$ if it has length less than $n$; analogously for $\mathrm{T}_{\mathrm{S} 5^{\mu}}^{2}$. By using the definition of negation we can get different formulations of the inference rules above, such as,

$$
\frac{\neg \Gamma, \alpha}{\neg \square \Gamma, \square \alpha, \Sigma}(\square) \quad \text { or } \quad \frac{\Gamma, \neg \alpha^{2}(\top)}{\Gamma, \neg v x . \alpha}\left(\mu^{2}\right) \quad \text { or } \quad \frac{\Gamma, \neg \alpha^{2}(\perp)}{\Gamma, \neg \mu x . \alpha}\left(v^{2}\right) .
$$

Note, that in the case of $\mathrm{T}_{\mathrm{S} 5^{\mu}}^{2}$, since we have an analytical cut rule, the search space for finding a proof of a given sequent is finite. Therefore, provability in $\mathrm{T}_{\mathrm{S} 5^{\mu}}^{2}$ is decidable.

\section{Correctness and more for $\mathbf{T}_{\mathrm{S}^{\mu}}^{\mathbf{1}}$}

PRoposition 4.1 (Correctness)

For all sequents $\Gamma \subset \mathcal{L}_{\mu}$ we have that

$$
\mathrm{T}_{\mathrm{S}^{\mu}}^{1} \vdash \Gamma \quad \Longrightarrow \quad=\mathrm{S} 5 \Gamma
$$

Proof. By induction on the length of derivation $n$. We restrict ourselves to transition systems such that for all states $s, s^{\prime}$ we have that $s \rightarrow s^{\prime}$ and $s^{\prime} \rightarrow s$. This is an admissible restriction since this is the case for all states $s, s^{\prime}$ where $s^{\prime}$ is reachable from $s$, and since validity in a state depends only on the reachable part (including the state itself) of the transition system. The base cases of the induction are trivial. For the induction step we prove only the case where the last inference rule was $\left(\right.$ ind $\left.^{+}\right)$. In this case we have that $\Gamma$ is of the form $\diamond \Delta, \square \Delta^{\prime}, \neg \varphi, v x . \alpha, \Sigma$ and we have that

$$
\mathrm{T}_{\mathrm{S} 5^{\mu}}^{1} \vdash^{<n} \diamond \Delta, \square \Delta^{\prime}, \neg \varphi, \alpha(\varphi) .
$$


By induction hypothesis for all S5-models $\mathcal{T}$ we have that

$$
\mathcal{T} \models \diamond \Delta, \square \Delta^{\prime}, \neg \varphi, \alpha(\varphi) .
$$

Let $s$ be a state in $\mathcal{T}$. If $s \models \diamond \Delta, \square \Delta^{\prime}$ then we trivially have $s \models \Gamma$. If this is not the case then it can easily be seen that since the reachability relation is an equivalence relation for all $s^{\prime}$ which are reachable from $s$ we have $s^{\prime}=\varphi \rightarrow \alpha(\varphi)$. Therefore we have that

$$
\mathcal{T}=\varphi \rightarrow \alpha(\varphi)
$$

But then $\|\varphi\|_{\mathcal{T}} \subseteq\|\alpha(\varphi)\|_{\mathcal{T}}$ and by definition of $\|v x . \alpha\|_{\mathcal{T}}$ we get $\|\varphi\|_{\mathcal{T}} \subseteq\|v x . \alpha\|_{\mathcal{T}}$ and therefore we get that $\mathcal{T} \models \Gamma$.

In the remaining part of this section we prove that for well-bounded and guarded formulae $v x . \alpha$ we have that $\mathrm{T}_{\mathrm{S} 5^{\mu}}^{1} \vdash v x . \alpha \leftrightarrow \alpha^{2}(\mathrm{~T})$ and that if $x$ is not guarded in $\alpha$ then we have that $\mathrm{T}_{\mathrm{S} 5^{\mu}}^{1} \vdash$ $\alpha(T) \leftrightarrow v x . \alpha$. We first show some structural properties of $\mathrm{T}_{\mathrm{S} 5^{\mu}}^{1}$. The weakening lemma is proved by a straightforward induction on the length of derivation.

LEMMA 4.2 (Weakening)

For all sequents $\Gamma, \Delta$ we have that

$$
\mathrm{T}_{\mathrm{S}^{\mu}}^{1} \vdash \Gamma \Longrightarrow \mathrm{T}_{\mathrm{S}^{\mu}}^{1} \vdash \Gamma, \Delta .
$$

The following lemma states some basic properties of $\mathrm{T}_{\mathrm{S}^{\mu}}^{1}$. The proof is left to the reader.

LEMMA 4.3

The following facts hold

(1) For all $\varphi$ we have $\mathrm{T}_{\mathrm{S} 5^{\mu}}^{1} \vdash \neg \varphi, \varphi$.

(2) $\mathrm{T}_{\mathrm{S}{ }^{\mu}}^{1} \vdash \Gamma, \sigma x . \alpha \Longleftrightarrow \mathrm{T}_{\mathrm{S}^{\mu}}^{1} \vdash \Gamma, \alpha(\sigma x . \alpha)$ where $\sigma \in\{\mu, \nu\}$.

(3) $\mathrm{T}_{\mathrm{S} 5^{\mu}}^{1} \vdash \Gamma \Longrightarrow \mathrm{T}_{\mathrm{S} 5^{\mu}}^{1} \vdash \Gamma[x / \varphi]$ for all $\varphi$.

(4) If $x$ appears positively in $\alpha(x)$ then from $\mathrm{T}_{\mathrm{S}^{\mu}{ }^{\mu}}^{1} \vdash \Gamma, \alpha(\beta)$ and $\mathrm{T}_{\mathrm{S}^{\mu}{ }^{\mu}}^{1} \vdash \neg \beta, \gamma$ we infer $\mathrm{T}_{\mathrm{S}^{\mu}{ }^{\mu}}^{1} \vdash$ $\Gamma, \alpha(\gamma)$.

\section{LEMMA 4.4}

The following facts hold

(1) $\mathrm{T}_{\mathrm{S} 5{ }^{\mu}}^{1} \vdash \neg \sigma x . \alpha(x, x), \sigma x . \sigma y . \alpha(x, y)$ where $\sigma \in\{\nu, \mu\}$.

(2) $\mathrm{T}_{\mathrm{S} 5^{\mu}}^{1} \vdash \sigma x . \alpha(x, x), \neg \sigma x . \sigma y . \alpha(x, y)$ where $\sigma \in\{v, \mu\}$.

Proof. Note, that if we prove both parts for the case where $\sigma=v$ then the case where $\sigma=\mu$ follows by definition of negation, indeed, part (1) follows from part (2) and vice versa.

For part (1) and $\sigma=v$ observe that by part (1) in Lemma $4.3 \neg \alpha(v x . \alpha, v x . \alpha), \alpha(v x . \alpha, v x . \alpha)$ is provable and by rule $\left(\right.$ unf $\left.^{\mu}\right)$ we get that the sequent $\neg v x . \alpha, \alpha(v x . \alpha, v x . \alpha)$ is provable, too. Applying twice the rule $\left(\right.$ ind $^{+}$) leads to the first part.

For part (2) in observe that by parts (1) and (2), Lemma 4.3 the sequent

$$
\neg v x . v y . \alpha(x, y), v y . \alpha(v x . v y . \alpha(x, y), y)
$$

is provable. Define $\psi: \equiv v y . \alpha(v x . v y . \alpha(x, y), y)$ then, by parts (1) and (2), in Lemma 4.3, we have that $\neg \psi, \alpha(v x . v y . \alpha(x, y), \psi)$ is provable. By applying this sequent and Equation (1) to part (4) in 
Lemma 4.3, we get that $\mathrm{T}_{\mathrm{S} 5^{\mu}}^{1} \vdash \neg \psi, \alpha(\psi, \psi)$ and with (ind ${ }^{+}$) we get

$$
\mathrm{T}_{\mathrm{S}^{\mu}}^{1} \vdash \neg \psi, v x . \alpha(x, x) .
$$

With Equation (1) and (cut) we get the result.

\section{Proposition 4.5}

For all formulae $\varphi \in \mathcal{L}_{\mu}$ we have that

$$
\mathrm{T}_{\mathrm{S}^{\mu}}^{1} \vdash \varphi \leftrightarrow \mathrm{wb}(\alpha)
$$

Proof. By formula structure of $\varphi$. The base cases where $\varphi$ is a propositional variable $p$ of $\sim p$ are clear. If $\varphi$ is of the form $\alpha \wedge \beta, \alpha \vee \beta, \square \alpha$ or $\diamond \alpha$ then the induction steps are straightforward. If $\varphi$ is of the form $v x . \alpha(x, \ldots, x)$ then by Lemma 4.4 we have that $v x . \alpha(x, \ldots, x) \leftrightarrow v x_{1} \ldots . v x_{n} . \alpha\left(x_{1}, \ldots, x_{n}\right)$ is provable and we get the induction step. Similarly for $\varphi$ of the form $\mu x . \alpha$.

In order to prove the next lemma we define a measure, $m(x, \varphi(x))$, for the complexity of $\varphi$ relative to $x$. Given a formula $\varphi(x)$ and a variable $x$ we define $m(x, \varphi(x))$ such that

- $m(x, \varphi)=0$ if $x \notin$ free $(\varphi)$,

- $m(x, x)=m(x, \sim x)=0$,

- $m(x, \alpha \circ \beta)=\max (m(x, \alpha), m(x, \beta))+1$ where $\circ \in\{\wedge, \vee\}$ and

- $m(x, \Delta \alpha)=m(x, \sigma y . \alpha)=m(x, \alpha)+1$ where $\Delta \in\{\square, \diamond\}$ and $\sigma \in\{\mu, \nu\}$.

LEMMA 4.6

The following facts hold

(1) For any formula $\varphi(x)$ such that $x \notin$ free $(\alpha, \beta)$ and such that $x$ appears only positively in $\varphi$ we have that

$$
\mathrm{T}_{\mathrm{S}^{\mu}{ }^{\mu}}^{1} \vdash \diamond \Delta, \square \Gamma, \neg \alpha, \beta \Longrightarrow \mathrm{T}_{\mathrm{S}^{\mu}{ }^{\mu}}^{1} \vdash \diamond \Delta, \square \Gamma, \neg \varphi(\alpha), \varphi(\beta) .
$$

(2) If $x$ appears guarded, positive and only once in $\alpha$ then we have

$$
\mathrm{T}_{\mathrm{S} 5^{\mu}}^{1} \vdash \neg \alpha^{2}(\top), \alpha^{3}(\top) .
$$

PROOF. The first part is proved by induction on $m(x, \varphi(x))$. If $m(x, \varphi(x))=0$ then either $\varphi \equiv x$ or $x \notin \operatorname{free}(\varphi)$. If $\varphi \equiv x$ then the implication of the claim is trivially true. If $x \notin$ free $(\varphi)$ then the claim follows by part (1) in Lemma 4.3. If $m(x, \varphi)>0$ then $\varphi$ is of the form $\gamma \wedge \delta, \gamma \vee \delta, \square \gamma, \diamond \gamma, \mu y \cdot \gamma(x, y)$ or $v y \cdot \gamma(x, y)$. We prove the case where $\varphi$ is of the form $\nu y \cdot \gamma(x, y)$. The case where $\varphi$ is of the form $\mu y \cdot \gamma(x, y)$ is dual and all the other cases are a straightforward induction.

So, let $\varphi$ be of the form $v y \cdot \gamma(x, y)$. Note, that for all $\alpha$ such that $x \notin \operatorname{free}(\alpha, \beta)$ we have that $m(x, \gamma(x, v y \cdot \gamma(\alpha, y)))<m(x, \nu y \cdot \gamma(x, y))$. Therefore, if we assume that $\mathrm{T}_{\mathrm{S} 5}^{1}{ }^{\mu} \vdash \diamond \Delta, \square \Gamma, \neg \alpha, \beta$ by induction hypothesis we can infer

$$
\mathrm{T}_{\mathrm{S} 5^{\mu}}^{1} \vdash \diamond \Delta, \square \Gamma, \neg \gamma(\alpha, v y \cdot \gamma(\alpha, y)), \gamma(\beta, v y \cdot \gamma(\alpha, y)) .
$$

An application of $\left(\right.$ unf $\left.^{\mu}\right)$ gives us that $\diamond \Delta, \square \Gamma, \neg v y \cdot \gamma(\alpha, y), \gamma(\beta, v y \cdot \gamma(\alpha, y))$ is provable and with $\left(\right.$ ind $^{+}$) we get the induction step

$$
\mathrm{T}_{\mathrm{S}^{\mu}}^{1} \vdash \diamond \Delta, \square \Gamma, \neg v y \cdot \gamma(\alpha, y), \nu y \cdot \gamma(\beta, y) .
$$


In order to prove that second part assume that $\alpha(x)$ is of the form $\beta(\Delta \gamma(x))$ where $\Delta \in\{\square, \diamond\}$. Further, note that $\alpha(T) \equiv \beta(\Delta \gamma(T))$. By part (1) in Lemma 4.3, we have that

$$
\mathrm{T}_{\mathrm{S}^{\mu}}^{1} \vdash \neg \Delta \gamma(\beta(\Delta \gamma(\mathrm{T}))), \Delta \gamma(\beta(\Delta \gamma(\mathrm{T}))), \neg \Delta \gamma(\mathrm{T})
$$

and with part (1) where $\varphi \equiv \Delta \gamma(\beta(x))$ we get that

$$
\mathrm{T}_{\mathrm{S}^{\mu}}^{1} \vdash \neg \Delta \gamma(\beta(\Delta \gamma(\mathrm{T}))), \Delta \gamma(\beta(\Delta \gamma(\beta(\Delta \gamma(\mathrm{T}))))), \neg \Delta \gamma(\beta(\Delta \gamma(\mathrm{T})))
$$

which means

$$
\mathrm{T}_{\mathrm{S}^{\mu}}^{1} \vdash \neg \Delta \gamma(\beta(\Delta \gamma(\mathrm{T}))), \Delta \gamma(\beta(\Delta \gamma(\beta(\Delta \gamma(\mathrm{T})))))
$$

and by applying part (1) where $\varphi \equiv \beta(x)$ again we have that

$$
\mathrm{T}_{\mathrm{S}^{\mu}}^{1} \vdash \neg \beta(\triangle \gamma(\beta(\Delta \gamma(\mathrm{T})))), \beta(\Delta \gamma(\beta(\triangle \gamma(\beta(\Delta \gamma(\mathrm{T}))))))
$$

which ends the proof of part (2).

The next theorem shows that for certain formulae the fixpoints are reached after two iterations and, therefore, provides a purely syntactical proof of a result which was proven with game theoretical methods in the joint work with Facchini [2].

\section{THEOREM 4.7}

If $x$ appears guarded, positive and only once in $\alpha(x)$ then we have that

(1) $\mathrm{T}_{\mathrm{S} 5^{\mu}}^{1} \vdash\left(\alpha^{2}(\mathrm{~T}) \leftrightarrow v x . \alpha\right) \wedge\left(\alpha^{2}(\perp) \leftrightarrow \mu x . \alpha\right)$, and

(2) $\models \mathrm{S} 5\left(\alpha^{2}(\top) \leftrightarrow v x . \alpha\right) \wedge\left(\alpha^{2}(\perp) \leftrightarrow \mu x . \alpha\right)$.

Proof. For part (1) the fact that $\alpha^{2}(\top) \rightarrow v x . \alpha$ is an easy consequence of part (2) in Lemma 4.6. The converse direction follows from the fact that $\alpha^{2}(v x . \alpha) \leftarrow v x . \alpha$ is provable and from part (4) in Lemma 4.3. The provability of $\alpha^{2}(\perp) \leftrightarrow \mu x . \alpha$ follows from the provability of $\alpha^{2}(\top) \leftrightarrow v x . \alpha$ and from definition of negation. Part (2) follows from Proposition 4.1 and part (1).

Let us end the section by proving that not guarded fixpoints are reached after one iteration.

\section{LEMMA 4.8}

If $x$ appears not guarded, positively and only once in $\varphi \in \mathcal{L}_{\mu}$, and if $\varphi$ is well-bounded then we have that

(1) $\mathrm{T}_{\mathrm{S} 5^{\mu}}^{1} \vdash \varphi(\mathrm{T}) \leftrightarrow v x . \varphi$,

(2) $\mathrm{T}_{\mathrm{S} 5^{\mu}}^{1} \vdash \varphi(\perp) \leftrightarrow \mu x . \varphi$ and

(3) $\mathrm{T}_{\mathrm{S}^{\mu}}^{1} \vdash(\alpha \rightarrow \beta) \rightarrow(\varphi(\alpha) \rightarrow \varphi(\beta))$.

Proof. We first prove that part (3) implies part (1). The proof of the fact that part (1) implies part (2) is left to the reader. In order to see that part (3) implies part (1) first observe that $T_{S 5^{\mu}}^{1} \vdash v x . \varphi \rightarrow \varphi(T)$ is a consequence of part (4) in Lemma 4.3 and of the fact that $\nu x . \varphi \rightarrow \varphi(v x . \varphi)$ is provable. In order to show the other implication we assume that we have part (1) for $\varphi$ and arbitrary $\alpha, \beta$. Set $\alpha \equiv \top$ and $\beta \equiv \varphi(\top)$. Then from part (1) we get

$$
\mathrm{T}_{\mathrm{S} 5^{\mu}}^{1} \vdash(\top \rightarrow \varphi(\top)) \rightarrow(\varphi(\top) \rightarrow \varphi(\varphi(\top))) .
$$


By some classical propositional reasoning we get that

$$
(\top \rightarrow \varphi(\top)) \rightarrow(\varphi(\top) \rightarrow \varphi(\varphi(\top)))
$$

is equivalent to $\varphi(\top) \rightarrow \varphi(\varphi(\top))$ and an application of (ind ${ }^{+}$) gives part (1).

It remains to prove part (3). This is done by induction on $\operatorname{rank}(\varphi)$. Note, that for the induction hypothesis we can use the statements of parts (1) and (2). The base cases are where $\varphi$ is the propositional variable $x$ or a variable $p$ are trivial. The induction steps for $\varphi$ of the form $\gamma \wedge \delta, \gamma \vee \delta$ are straightforward.

If $\varphi$ is of the form $\square \gamma$ or $\diamond \gamma$ then since $x$ is not guarded in $\varphi$ we have that $x \notin$ free $(\gamma)$ and the claim of part (3) is trivial.

If $\varphi$ is of the form $v y \cdot \gamma(x, y)$ then we distinguish two cases. In the first case $y$ is not guarded in $\gamma$. Then, by induction hypothesis for all $\alpha, \beta$ have that

$$
\mathrm{T}_{\mathrm{S} 5^{\mu}}^{1} \vdash(\alpha \rightarrow \beta) \rightarrow(\gamma(\alpha, \top) \rightarrow \gamma(\beta, \top)) .
$$

By induction hypothesis for part (1) we get that

$$
\mathrm{T}_{\mathrm{S} 5^{\mu}}^{1} \vdash \gamma(x, \mathrm{\top}) \leftrightarrow v y \cdot \gamma(x, y)
$$

and with part (3) in Lemma 4.3 we get that

$$
\mathrm{T}_{\mathrm{S}^{\mu}}^{1} \vdash \gamma(\alpha, \mathrm{T}) \leftrightarrow v y \cdot \gamma(\alpha, y) \text { and } \mathrm{T}_{\mathrm{S}^{\mu}}^{1} \vdash \gamma(\beta, \mathrm{T}) \leftrightarrow \nu y \cdot \gamma(\beta, y)
$$

Two applications of part (4) in Lemma 4.3 to Equation (2) give us the induction step. In the second case, we have that $y$ is guarded in $\gamma$. The induction step goes similarly by using the fact that by induction hypothesis we have that

$$
\mathrm{T}_{\mathrm{S}^{\mu}}^{1} \vdash(\alpha \rightarrow \beta) \rightarrow(\gamma(\alpha, \gamma(\alpha, \mathrm{T})) \rightarrow \gamma(\beta, \gamma(\beta, \mathrm{T})))
$$

and that from Theorem 4.7 , since $v y \cdot \gamma$ is assumed to be well-bounded, we have that

$$
\mathrm{T}_{\mathrm{S}^{\mu}{ }^{\mu}}^{1} \vdash \gamma(\alpha, \gamma(\alpha, \mathrm{T})) \leftrightarrow \nu y \cdot \gamma(\alpha, y) \text { and } \mathrm{T}_{\mathrm{S}^{\mu}}^{1} \vdash \gamma(\beta, \gamma(\beta, \mathrm{T})) \leftrightarrow \nu y \cdot \gamma(\beta, y) .
$$

The case where $\varphi$ is of the form $\mu x . \gamma$ is proven similarly as the case where $\varphi$ is of the form $\nu x . \gamma$.

COROLlary 4.9

If $x$ appears not guarded, positive and only once in $\varphi(x) \in \mathcal{L}_{\mu}$, and if $\varphi$ is well-bounded then we have that

$$
\models \mathrm{S} 5(v x . \varphi \leftrightarrow \varphi(\top)) \wedge(\mu x . \varphi \leftrightarrow \varphi(\perp))
$$

\section{Completeness of $\mathbf{T}_{\mathrm{S} 5^{\mu}}^{2}$}

In this section, we prove completeness for well-bounded of $\mathrm{T}_{\mathrm{S} 5^{\mu}}^{2}$. We start with a lemma showing some basic properties of $\mathrm{T}_{\mathrm{S} 5^{\mu}}^{2}$. The proof is left to the reader. 


\section{LEMMA 5.1}

For all formulae $\alpha$ and sets of formulae $\Gamma, \Delta$ we have that

(1) If $\mathrm{T}_{\mathrm{S}^{\mu}}^{2} \vdash \Gamma$ then $\mathrm{T}_{\mathrm{S}^{\mu}{ }^{\mu}}^{2} \vdash \Gamma, \Delta$,

(2) $\mathrm{T}_{\mathrm{S}_{5} \mu}^{2} \vdash \alpha, \neg \alpha$,

(3) $\mathrm{T}_{\mathrm{S} 5^{\mu}}^{2} \vdash \neg \square \alpha, \alpha, \mathrm{T}_{\mathrm{S} 5^{\mu}}^{2} \vdash \neg \square \alpha, \square \square \alpha$, and $\mathrm{T}_{\mathrm{S} 5^{\mu}}^{2} \vdash \neg \vee \alpha, \square \triangleright \alpha$.

In order to prove completeness we need some well-known notions: a set of formulae $\Gamma$ is called consistent if for all finite subsets $\Gamma^{\prime} \subseteq \Gamma$ we have that $\mathrm{T}_{\mathrm{S} 5^{\mu}}^{2} \not \neg \neg \Gamma^{\prime}$. It is maximal consistent if for all formulae $\alpha$ such that $\Gamma, \alpha$ is consistent we have that $\alpha \in \Gamma$. The canonical model for a formula $\varphi, \mathcal{M}_{\varphi}$, is defined such that the set of states is

$$
\{M \cap \mathcal{C}(\varphi) ; M \text { is maximal consistent and }\{\varphi, \neg \varphi\} \cap M \neq \emptyset\},
$$

for two states $M, M^{\prime}$ we have that $M \rightarrow M^{\prime}$ if $\{\alpha ; \square \alpha \in M\} \subseteq M^{\prime}$, and for all propositional variables $p \leq \varphi$ we have that $\lambda(p)=\{M ; p \in M\}$.

Note that by the part (1) in the following Lemma 5.2, we cannot have that a propositional variable $p$ and its negation $\sim p$ occur in the same maximal consistent set and that the valuation $\lambda$ is well-defined. The next lemma shows some basic properties of canonical models.

LEMMA 5.2

Let $\mathcal{M}_{\varphi}$ be a canonical model. For all states $M$ and all formulae $\alpha, \beta \in \mathcal{C}(\varphi)$ we have that

(1) $\alpha \in M \Leftrightarrow \neg \alpha \notin M$.

(2) If $\alpha \wedge \beta \in \mathcal{C}(\varphi)$ then: $\alpha \wedge \beta \in M \Leftrightarrow \alpha, \beta \in M$.

(3) If $\alpha \vee \beta \in \mathcal{C}(\varphi)$ then: $\alpha \vee \beta \in M \Leftrightarrow(\alpha \in M)$ or $(\beta \in M)$.

(4) $\alpha \in M$ and $\mathrm{T}_{\mathrm{S}^{\mu}{ }^{\mu}}^{2} \vdash \neg \alpha, \beta$ then $\beta \in M$.

PROOF. We prove only part (1). All other parts go through with similar arguments. First, we see that if $\alpha, \neg \alpha \in M$ then by definition of consistent set we have that $\mathrm{T}_{\mathrm{S} 5^{\mu}}^{1} \not \neg \neg \alpha, \alpha$ but by Lemma 5.1 this is not the case. Now, assume that there is an $\alpha \in \mathcal{C}(\varphi)$ such that $\alpha, \neg \alpha \notin M$ and assume that $\varphi \in M$ (instead of $\neg \varphi \in M$ ). We claim that either $M \cup\{\alpha\}$ or $M \cup\{\neg \alpha\}$ is consistent. For, this was not the case then we would have $\mathrm{T}_{\mathrm{S} 5^{\mu}}^{2} \vdash \neg M, \neg \alpha$ and $\mathrm{T}_{\mathrm{S}^{\mu}{ }^{\mu}}^{2} \vdash \neg M, \alpha$. But since $\varphi \in M$ and $\alpha \in \mathcal{C}(\varphi)$ by (Ccut) we have that

$$
\mathrm{T}_{\mathrm{S}^{\mu}}^{2} \vdash \neg M
$$

and, therefore, that $M$ is not consistent.

Proposition 5.3

For any formula $\varphi \in \mathcal{L}_{\mu}$ the canonical model $\mathcal{M}_{\varphi}$ is an $\mathrm{S} 5$ model, that is, the accessibility relation is reflexive, transitive and symmetric.

Proof. For reflexivity we have to show that for all states $M$ of $\mathcal{M}_{\varphi}$ we have that $\square \alpha \in M$ implies $\alpha \in M$. But this is a consequence of part (3) in Lemma 5.1 and part (4) in Lemma 5.2.

For transitivity we have to show that

$$
M \rightarrow M^{\prime} \text { and } M^{\prime} \rightarrow M^{\prime \prime} \Longrightarrow\left(\square \alpha \in M \Longrightarrow \alpha \in M^{\prime \prime}\right) .
$$

Assume that $\square \alpha \in M$. We distinguish two cases. In the first case we have that $\square \square \alpha \in \mathcal{C}(\varphi)$. Then, since by part (3) in Lemma 5.1 we have that

$$
\mathrm{T}_{\mathrm{S}^{\mu}}^{2} \vdash \neg \square \alpha, \square \square \alpha
$$


with part (4) in Lemma 5.2 we get that $\square \square \alpha \in M$, and by construction also $\alpha \in M^{\prime \prime}$. In the second case we have that $\square \square \alpha \notin \mathcal{C}(\varphi)$. Then, by construction of $\mathcal{C}(\varphi)$ we have that $\alpha$ is either of the form $\square \beta$ or of the form $\diamond \beta$. In the first case, we have that $\square \beta \in M^{\prime}$ and since we have Equation (3) also for $\beta$ we get that $\square \square \beta \in M^{\prime}$ and from that we get $\square \beta \in M^{\prime \prime}$. In the latter case we that $\diamond \beta \in M^{\prime}$. Since by part (3) in Lemma 5.1 we have

$$
\mathrm{T}_{\mathrm{S}^{\mu}}^{2} \vdash \neg \diamond \beta, \square \diamond \beta
$$

with similar arguments we get that $\diamond \beta \in M^{\prime \prime}$.

For the symmetry we have to show that

$$
M \rightarrow M^{\prime} \Longrightarrow\left(\square \alpha \in M^{\prime} \Longrightarrow \alpha \in M\right)
$$

Assume the contrapositive, i.e. $M \rightarrow M^{\prime}$ and $\square \alpha \in M^{\prime}$ and $\neg \alpha \in M$. Then, by part (3) in Lemma 5.1 and part (4) in Lemma 5.2, we have that $\neg \square \alpha \in M$. Again we distinguish following two cases.

If $\square \neg \square \alpha \in \mathcal{C}(\varphi)$, then, since by part (3) in Lemma 5.1 we have that $\mathrm{T}_{\mathrm{S} 5^{\mu}}^{2} \vdash \square \alpha, \square \neg \square \alpha$ by part (4) in Lemma 5.2 we get that $\square \neg \square \alpha \in M$ and by construction that $\neg \square \alpha \in M^{\prime}$, which is a contradiction.

If $\square \neg \square \alpha \notin \mathcal{C}(\varphi)$ then by construction $\alpha$ is of the form $\square \beta$ or $\diamond \beta$. In the former case we have that $\neg \square \beta \in M$ but then $\square \neg \square \beta \in M$ and, therefore, $\neg \square \beta \in M^{\prime}$, which is a contradiction. In the latter case we have that $\neg \diamond \beta \in M$ but then $\square \neg \diamond \beta \in M$ and, therefore, $\neg \diamond \beta \in M^{\prime}$, which is a contradiction, too.

LEMMA 5.4

Let $\varphi$ be a well-bounded formula. For all formulae $\alpha \leq \varphi$ and all states $M$ of the canonical model $\mathcal{M}_{\varphi}$ we have that

$$
\alpha \in M \quad \Longrightarrow \quad M \models \alpha .
$$

Proof. By induction on the rank of $\alpha$. Note, that by Lemma 3.1 we have that all $\alpha \in M$ are wellbounded. The cases where $\alpha$ is of the form $p, \sim p, \beta \wedge \gamma, \beta \vee \gamma$ go through straightforwardly with Lemma 5.2. The cases where $\alpha$ is of the form $\diamond \beta$ or $\square \beta$ go through with standard arguments.

If $\alpha$ is of the form $\mu x$. $\beta$ since $\alpha$ is well-bounded we have that $x$ has at most one free occurrence in $\beta$. If $x$ appears guarded in $\beta$ then note that by part (2) in Lemma 5.1 we have that $\mathrm{T}_{\mathrm{S} 5^{\mu}}^{2} \vdash \neg \beta^{2}(\perp), \beta^{2}(\perp)$ and one application of $\left(\nu^{2}\right)$ yields $\mathrm{T}_{\mathrm{S} 5^{\mu}}^{2} \vdash \neg \mu x . \beta, \beta^{2}(\perp)$ and, therefore by part (4) in Lemma 5.2, that $\beta^{2}(\perp) \in M$. We can apply the induction hypothesis and we get that $M \models \beta^{2}(\perp)$. With Theorem 4.7 we get that

$$
M \models \mu x . \beta .
$$

The case where $x$ is not guarded in $\beta$ goes similarly and if $\alpha$ is of the form $v x$. $\beta$ then we also use similar arguments.

THEOREM 5.5

For all well-bounded formulae $\varphi \in \mathcal{L}_{\mu}$ we have that

$$
\vDash \mathrm{S} 5 \varphi \quad \Longrightarrow \quad \mathrm{T}_{\mathrm{S}^{\mu}}^{2} \vdash \varphi .
$$

ProOF. We prove the contrapositive. If we have that $\mathrm{T}_{\mathrm{S} 5^{\mu}}^{2} \forall \varphi$ then $\neg \varphi$ is consistent and can be extended to a maximal consistent set. Therefore, in the canonical model $\mathcal{M}_{\varphi}$ there is a state $M$ such that $\neg \varphi \in M$. Since $\neg \varphi$ is also well-bounded by Lemma 5.4 we have that $M \models \neg \varphi$ and, therefore, that $\not \subset \mathrm{S} 5 \varphi$. 


\section{Completeness and correctness of $\mathrm{T}_{\mathrm{S} 5^{\mu}}^{1}$ and $\mathrm{T}_{\mathrm{S} 5^{\mu}}^{2}$}

\section{LEMMA 6.1}

For all sequents $\Gamma$ we have that

$$
\mathrm{T}_{\mathrm{S}^{\mu}{ }^{\mu}}^{2} \vdash \mathrm{wb}(\Gamma) \Rightarrow \mathrm{T}_{\mathrm{S}^{\mu}{ }^{\mu}}^{1} \vdash \Gamma .
$$

Proof. By Proposition 4.5 we equivalently can show that

$$
\mathrm{T}_{\mathrm{S} 5^{\mu}}^{2} \vdash \mathrm{wb}(\Gamma) \Rightarrow \mathrm{T}_{\mathrm{S}^{\mu}}^{1} \vdash \mathrm{wb}(\Gamma) .
$$

This is shown by induction on the proof length $n$ of $\mathrm{T}_{\mathrm{S}^{\mu}}^{2} \vdash \mathrm{wb}(\Gamma)$. The case where $n=0$ is clear. If $n>0$ the induction step goes by case distinction on the last inference rule. All cases except the case where the last inference rule was $\left(v^{2}\right),\left(\mu^{2}\right),(\mu),(v)$ are straightforward. For the case where it was $\left(\mu^{2}\right)$ we have that $\mathrm{wb}(\Gamma)$ is of the form $\Delta, v x . \varphi(x)$ and that

$$
\mathrm{T}_{\mathrm{S}^{\mu}{ }^{\mu}}^{2} \vdash^{<n} \Delta, \varphi^{2}(\mathrm{~T})
$$

By induction hypothesis we have that $T_{S 5^{\mu}}^{1} \vdash \Delta, \varphi^{2}(\top)$. Since $x$ appears guarded and at most once in $\varphi(x)$ by part (2) in Lemma 4.6 we have that $\mathrm{T}_{\mathrm{S}^{\mu}}^{1} \vdash \neg \varphi^{2}(\top), \varphi^{3}(\top)$ and, therefore, with (ind ${ }^{+}$) we get that $\mathrm{T}_{\mathrm{S} 5^{\mu}}^{1} \vdash \neg \varphi^{2}(\top), v x . \varphi$. With cut we get the desired result. The case for $\left(v^{2}\right)$ goes similar. The cases for $(\mu),(v)$ use Lemma 4.8 and are analogous.

Combining Lemma 6.1 with Theorem 5.5 and Proposition 4.1 yields the following theorem.

THEOREM 6.2 (Completeness and correctness)

Let $\Gamma$ be any sequent. We have that

$$
\models \mathrm{S} 5 \Gamma \quad \Leftrightarrow \quad \mathrm{T}_{\mathrm{S}^{\mu}}^{2} \vdash \mathrm{wb}(\Gamma) \Leftrightarrow \mathrm{T}_{\mathrm{S}^{\mu}{ }^{\mu}}^{1} \vdash \Gamma .
$$

\section{Conclusion: completeness of $\mathrm{Koz}^{\mathrm{S} 5}$}

We first define a translation $t: \mathcal{L}_{\mu}^{\mathrm{wb}} \rightarrow \mathcal{L}_{\text {mod }}$ from the class of well-bounded $\mu$-formulae to the modal fragment recursively such that $t(p) \equiv p$ and $t(\sim p) \equiv \sim p$, such that $t$ distributes over boolean and modal connectives and such that:

- If $x$ appears guarded in $\alpha$ then

$$
t(\mu x . \alpha) \equiv t(\alpha)[x / t(\alpha)[x / \perp]] \text { and } t(v x . \alpha) \equiv t(\alpha)[x / t(\alpha)[x / \top]]
$$

- If $x$ is not guarded in $\alpha$ then

$$
t(\mu x . \alpha) \equiv t(\alpha)[x / \perp] \text { and } t(v x . \alpha) \equiv t(\alpha)[x / \top] .
$$

The fact that the definition of $t$ terminates follows from the in the defining clauses rank decreases and the formula remains well-bounded. Further, note that we have that $t(\varphi) \in \mathcal{L}_{\text {mod }}$. 


\section{Sequent Calculi for the Modal $\mu$-Calculus over S5}

\section{LEMMA 7.1}

For all well-bounded formulae $\varphi$ we have that

$$
\mathrm{Koz}^{\mathrm{S} 5} \vdash \varphi \leftrightarrow t(\varphi)
$$

Proof. By induction on $\operatorname{rank}(\varphi)$. In the proof we abbreviate $t(\alpha)[x / t(\alpha)]$ by $(t(\alpha))^{2}$, and analogously for $(t(\alpha))^{3}$. The base cases of the induction are trivial and the induction steps where the formula $\varphi$ is of the from $\alpha \wedge \beta, \alpha \vee \beta, \square \alpha$ or $\vee \alpha$ are straightforward. If $\varphi$ is of the form $\nu x . \alpha$ and $x$ is guarded in $\alpha$ then, since $t(\alpha) \in \mathcal{L}_{\text {mod }}$, by Proposition 2.2 and Theorem 4.7, we have that

$$
\mathrm{Koz}^{\mathrm{S} 5} \vdash(t(\alpha))^{2}[x / \top] \leftrightarrow(t(\alpha))^{3}[x / \top] .
$$

An application of (ind) yields Koz ${ }^{\mathrm{S} 5} \vdash(t(\alpha))^{2}[x / \top] \rightarrow v x . t(\alpha)$. And since also $v x . t(\alpha) \rightarrow(t(\alpha))^{2}[x / \top]$ is provable, we get

$$
\mathrm{Koz}^{\mathrm{S} 5} \vdash(t(\alpha))^{2}[x / \top] \leftrightarrow v x . t(\alpha)
$$

Since by induction hypothesis we have that $\mathrm{Koz}^{\mathrm{S} 5} \vdash \alpha \leftrightarrow t(\alpha)$ we also can show that $\mathrm{Koz}^{\mathrm{S} 5} \vdash$ $v x . \alpha \leftrightarrow v x . t(\alpha)$ and, therefore, we get that

$$
\mathrm{Koz}^{\mathrm{S} 5} \vdash(t(\alpha))^{2}[x / \top] \leftrightarrow v x . \alpha .
$$

The induction step follows from the fact that $t(v x . \alpha) \equiv(t(\alpha))^{2}[x / \top]$. If $\varphi$ is of the form $v x . \alpha$ and $x$ is not guarded in $\alpha$ then the induction step follows by an analogous argument using the fact that by Proposition 2.2 and Corollary 4.9 we have that $\mathrm{Koz}^{\mathrm{S} 5} \vdash t(\alpha)[x / \top] \leftrightarrow(t(\alpha))^{2}[x / \top]$. The cases where $\varphi$ is of the form $\mu x . \alpha$ are analogous to the previous cases.

The next lemma is proved like Proposition 4.5 by using the fact that the proof of Lemma 4.4 goes through also with the normal induction rule (ind) instead of (ind ${ }^{+}$).

LEMMA 7.2

For all formulae $\varphi \in \mathcal{L}_{\mu}$ we have that

$$
\mathrm{Koz}^{\mathrm{S} 5} \vdash \varphi \leftrightarrow \mathrm{wb}(\varphi)
$$

THEOREM 7.3 (Completeness and correctness of $\mathrm{Koz}^{\mathrm{S} 5}$ )

For all formulae $\varphi \in \mathcal{L}_{\mu}$ we have that

$$
=\mathrm{S} 5 \varphi \quad \Leftrightarrow \quad \mathrm{Koz}^{\mathrm{S} 5} \vdash \varphi
$$

Proof. The correctness is Proposition 2.2. For the completeness note that by correctness, Lemma 7.1 and Lemma 7.2 we have that

$$
\models \mathrm{S} 5 \varphi \leftrightarrow t(\mathrm{wb}(\varphi)) .
$$

Therefore if $\models \mathrm{S} 5 \varphi$ then $\models \mathrm{S} 5 t(\mathrm{wb}(\varphi))$. Since $t(\mathrm{wb}(\varphi)) \in \mathcal{L}_{\text {mod }}$ by Proposition 2.2 we have that $\mathrm{Koz}^{\mathrm{S} 5} \vdash t(\mathrm{wb}(\varphi))$ and with Lemmas 7.2 and 7.1 we finish the proof.

CONCLUding REMARK. We have three crucial steps in the completeness proof of $\mathrm{Koz}^{\mathrm{S} 5}$. First, the completeness of $\mathrm{Koz}^{\mathrm{S} 5}$ over the modal fragment; second, the fact that $\mathrm{Koz}^{\mathrm{S} 5}$ proves the equivalence 
of $\varphi$ and $t(\mathrm{wb}(\varphi))$; and, third, that for guarded and well-bounded $\varphi$ we have that $\varphi^{2}(\top)$ and $\varphi^{3}(\top)$ are semantically equivalent (and analogously for not guarded formulae). As said in the introduction the third fact was shown in a joint work with Facchini [2] by using game-theoretical methods and the correspondence of parity games and modal $\mu$-calculus. Therefore, by using this game-theoretical result the completeness proof for $\mathrm{Koz}^{\mathrm{S} 5}$ would have been possible without the 'detour' via $\mathrm{T}_{\mathrm{S} 5}^{1}$ and $\mathrm{T}_{\mathrm{S} 5^{\mu}}^{2}$. Nevertheless, introducing $\mathrm{T}_{\mathrm{S} 5^{\mu}}^{1}$ and $\mathrm{T}_{\mathrm{S} 5^{\mu}}^{2}$ allowes us to give a purely proof-theoretically proof of this equivalence without using any connections to game-theory. Further, in the case of $\mathrm{T}_{\mathrm{S} 5^{\mu}}^{2}$, it allows us provide a calculus with analytical cut.

\section{Funding}

Hasler Foundation [Project-Nr.: 2192].

\section{References}

[1] L. Alberucci. A syntactical treatment of simultaneous fixpoints in the modal $\mu$-calculus. Technical Report IAM-09-001. University of Berne, 2009.

[2] L. Alberucci and A. Facchini. The modal $\mu$-calculus hierarchy on restricted classes of transition systems. Journal of Symbolic Logic (inpress).

[3] A. Arnold and D. Niwinski. Rudiments of Mu-calculus. Elsevier Science, North-Holland, 2001.

[4] B. Chellas. Modal Logic. Cambridge University Press, 1980.

[5] M. Dam and C. Sprenger. On the structure of inductive reasoning: circular and treeshaped proofs in the $\mu$-calculus. In Foundations of Software Science and Computational Structures: 6th International Conference. Vol. 2620 of the Lecture Notes in Computer Science. A. D. Gordon (ed.). pp. 425-440. Springer, 2003.

[6] G. Jäger, M. Kretz and T. Studer. Canonical completeness of infinitary mu. Journal of Logic and Algebraic Programming, 76, 270-292, 2008.

[7] D. Kozen. Results on the propositional mu-calculus. Theoretical Computer Science, 27, 333-354, 1983.

[8] T. Studer. On the proof theory of modal mu-calculus. Studia Logica, 89, 343-363, 2008.

[9] A. Tarski. A lattice-theoretical fixpoint theorem and its applications. Pacific Journal of Mathematics, 5, 285-309, 1955.

[10] I. Walukiewicz. Completeness of Kozen's axiomatisation of the propositional mu-calculus. Information and Computation, 157, 142-182, 2000.

Received 24 July 2008 